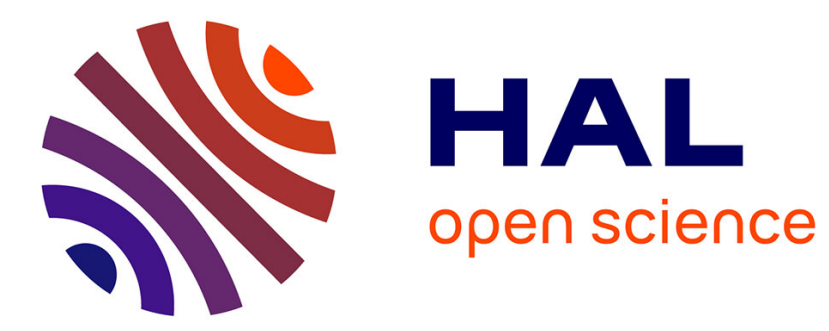

\title{
Absorption optimale par contrôle actif
}

\author{
D. Thenail, M. Galland, M. Sunyach
}

\section{To cite this version:}

D. Thenail, M. Galland, M. Sunyach. Absorption optimale par contrôle actif. Journal de Physique IV Proceedings, 1994, 04 (C5), pp.C5-195-C5-198. 10.1051/jp4:1994537 . jpa-00253032

\section{HAL Id: jpa-00253032 https://hal.science/jpa-00253032}

Submitted on 1 Jan 1994

HAL is a multi-disciplinary open access archive for the deposit and dissemination of scientific research documents, whether they are published or not. The documents may come from teaching and research institutions in France or abroad, or from public or private research centers.
L'archive ouverte pluridisciplinaire HAL, est destinée au dépôt et à la diffusion de documents scientifiques de niveau recherche, publiés ou non, émanant des établissements d'enseignement et de recherche français ou étrangers, des laboratoires publics ou privés. 


\title{
Absorption optimale par contrôle actif
}

\author{
D. THENAIL, M.A. GALLAND et M. SUNYACH
}

Laboratoire de Mécanique des Fluides et d'Acoustique, URA 263 du CNRS, Ecole Centrale de Lyon, UCB-Lyon I, BP. 163, 69131 Ecully cedex, France

\begin{abstract}
We present in this paper the use of active control techniques to enhance absorbent materials performances. The weak low frequency efficiency, which is usually observed even when particularly bulky amounts are used, is shown to be improved by an active support. We firstly determine the maximum absorption criteria, and present active control experimental results.
\end{abstract}

\section{INTRODUCTION}

Si l'efficacité des matériaux poreux est reconnue pour des gammes de moyennes et hautes fréquences, ils sont en revanche très peu absorbants dans les gammes de basses fréquences, même employés en de grandes épaisseurs.

Cette étude vise à optimiser les performances de ces matériaux dans ce dernier cas, à l'aide de techniques de contrôle actif. Dans un premier temps, on cherchera à déterminer les conditions permettant d'obtenir une absorption maximale, puis on les recréera artificiellement par un système actif approprié.

\section{MODELISATION}

\subsection{Position du problème}

On peut réaliser une absorption importante en très basses fréquences, en plaçant le matériau poreux à une distance égale au quart de la longueur d'onde d'une paroi rigide. Cette méthode largement utilisée est cependant approximative. Nous avons voulu, ici, déterminer de façon plus générale les conditions qui permettent, en incidence normale, d'obtenir un coefficient d'absorption égal à 1 . Le problème peut alors s'énoncer de la façon suivante : quelle est l'impédance $Z$ qu'on doit appliquer à la face arrière d'un échantillon de matériau poreux d'épaisseur d, pour que l'absorption soit totale ? (figure 1)

milieu poreux

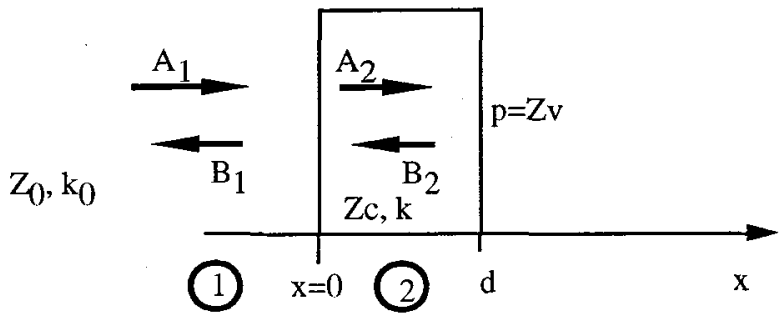

Fig. 1. - Matériau absorbant fermé sur une impédance.

\subsection{Modèle}

Pour résoudre ce problème, il faut se donner un modèle de propagation acoustique dans le milieu poreux. Les lois habituellement utilisées peuvent être empiriques (Delany - Bazley [1]) ou élaborées à partir de considérations sur les propriétés géométriques et physiques des matériaux ( Allard [2] ). Elles 
permettent d'obtenir l'impédance caractéristique $Z_{\mathfrak{c}}$ du milieu et le nombre d'onde complexe $\mathrm{k}$ comme fonction d'une quantité dépendant de la fréquence f d'excitation, et de l'imperméabilité $\sigma$ du matériau :

On peut alors résoudre le problème précédemment posé en calculant le coefficient de réflexion en amplitude $\mathrm{r}$ :

$$
r=\frac{B_{1}}{A_{1}}=\frac{(\zeta+\alpha)(\alpha-1)+(\zeta-\alpha)(\alpha+1) e^{-2 i k d}}{(\zeta+\alpha)(\alpha+1)+(\zeta-\alpha)(\alpha-1) e^{-2 i k d}} \text { où: } \zeta=\frac{Z}{Z_{0}} \text { et } \alpha=\frac{Z_{c}}{Z_{0}}, Z_{0} \text { impedance de l'air. }
$$

L'impédance réduite optimale est obtenue alors pour un coefficient de réflexion nul :

$$
\zeta_{\text {opt }}=\alpha \frac{(\alpha+1) e^{-2 i k d}-(\alpha-1)}{(\alpha+1) e^{-2 i k d}+(\alpha-1)}
$$

On a représenté sur la figure 2 l'évolution dans le plan complexe de cette impédance dans la bande des fréquences audibles, pour deux épaisseurs d'un matériau d'imperméabilité égale à $30000 \mathrm{Nm}^{-4} s$, ce qui correspond approximativement à la laine de verre utilisée dans nos expériences.

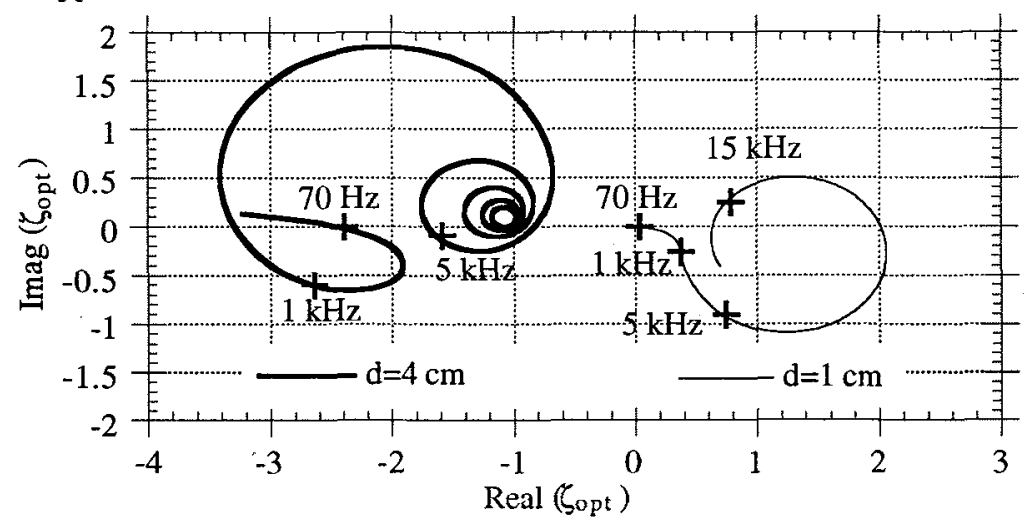

Fig. 2. - Impédance arrière optimale de deux échantillons de laine de verre

On constate sur cette figure que l'impédance optimale est totalement différente pour les deux épaisseurs. Ainsi, avec $1 \mathrm{~cm}$ de matériau, on retrouve approximativement pour les très basses fréquences la condition en $\lambda / 4$, qui correspond à une impédance arrière nulle. Ce n'est en revanche pas du tout le cas avec $4 \mathrm{~cm}$ de laine de verre.

\subsection{Validation expérimentale} figure 3.

On a cherché à retrouver expérimentalement ces résultats, en utilisant le dispositif représenté sur la

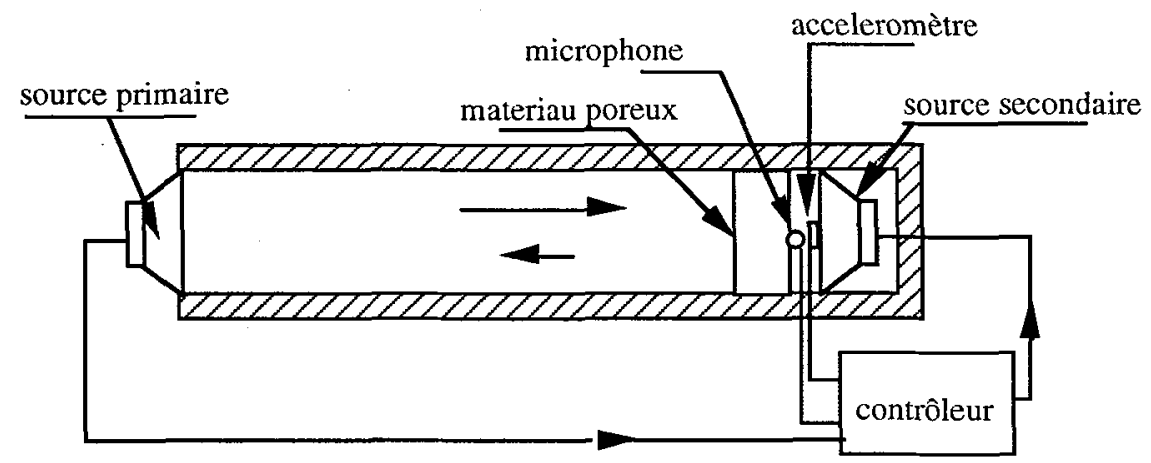

Fig. 3. - Dispositif expérimental.

La mesure du coefficient d'absorption est réalisée en tube de Kundt par la méthode à deux microphones de Chung et Blaser [3] . L'impédance arrière $Z$ est imposée par un système de contrôle actif comportant un haut-parleur à membrane plane, de surface émissive équivalente à la section du tube. $\mathrm{La}$ vitesse acoustique est alors mesurée à l'aide d'un accéléromètre, la pression étant obtenue à l'aide d'un microphone placé à proximité. Leur connaissance nous permet de fixer l'impédance terminale $Z$ (Thenail 
[4])grâce à un filtre de contrôle auto-adaptatif qui minimise l'énergie du signal d'erreur : $\varepsilon=p-Z v$. On

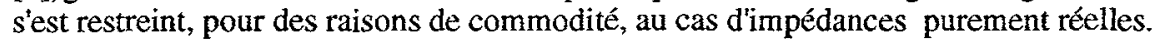

$\mathrm{La}$ fréquence d'excitation est de $70 \mathrm{~Hz}$ pour les deux échantillons de laine de verre. On a représenté sur la figure 4 les comparaisons entre les coefficients d'absorption théoriques et mesurés. L'accord excellent obtenu nous permet de conclure à la bonne représentativité du modèle.

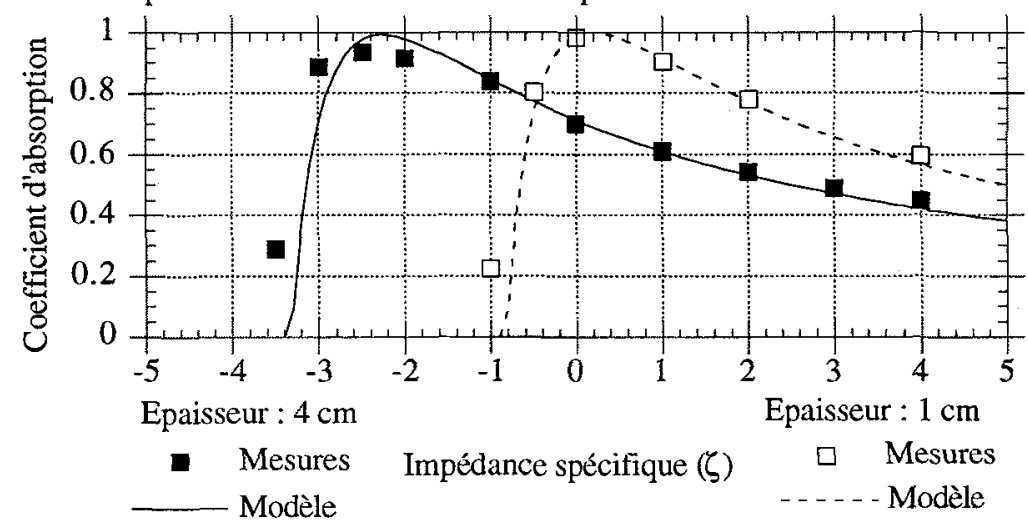

Fig. 4. - Variation du coefficient d'absorption d'un échantillon de laine de verre $(\mathrm{f}=70 \mathrm{~Hz})$ en fonction de son impédance arrière modifiée par contrôle actif.

\section{OPTIMISATION PAR CONTROLE ACTIF}

\subsection{Principe du contrôle d'absorption}

Le but du système de contrôle actif est ici d'imposer la condition d'absorption maximale, ou tout du moins, une condition approchée qui permette une mise en ouvre simplifiée. La réalisation d'une impédance donnée nécessite en effet la mesure de la vitesse acoustique et conduit à des réalisations expérimentales complexes. Or, les résultats de l'étude théorique nous ont prouvé que pour une épaisseur bien choisie de matériau, ici $1 \mathrm{~cm}$, l'optimum était atteint avec une impédance arrière proche de 0 , ce qui ramène alors le contrôle actif d'impédance à une classique minimisation de pression.

\subsection{Validation du principe}

Dans un premier temps, on a cherché à vérifier si le principe de contrôle retenu précédemment conduisait à une absorption conséquente. Les mesures ont été effectuées en bande large, dans le tube de Kundt décrit plus haut, jusquà $1400 \mathrm{~Hz}$, fréquence de coupure du conduit. Le système de contrôle placé à l'arrière de l'échantillon de $1 \mathrm{~cm}$ d'épaisseur, comprend désormais uniquement un microphone de contrôle. Dans ce premier essai, le contrôleur est un filtre numérique auto-adaptatif en temps réel, programmé selon l'algorithme L.M.S. à référence filtrée, classiquement utilisé en anti-bruit. Le signal de référence est le signal qui alimente la source primaire. On voit en figure 5 que le coefficient d'absorption, très bas lorsque la laine de verre est directement plaquée sur un fond rigide, prend des valeurs très proches de 1, lorsque le contrôle opère.

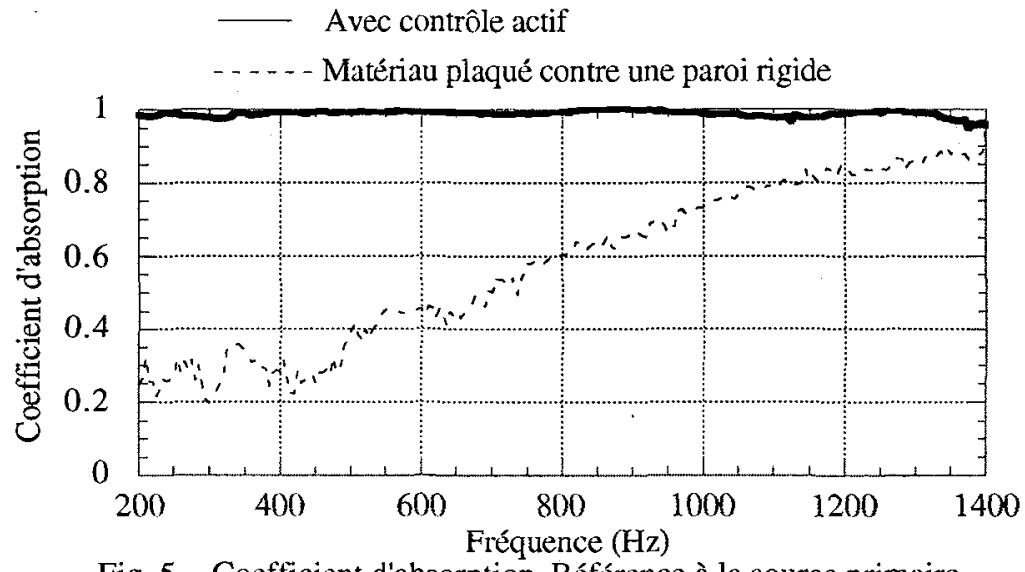

Fig. 5. - Coefficient d'absorption. Référence à la source primaire 


\subsection{Contrôle avec une référence locale.}

En général, on ne dispose pas du signal générant le bruit. Il faut alors concevoir un système de contrôle fonctionnant avec un signal de référence pris localement, par un microphone placé à proximité de l'absorbant. Nous avons ainsi testé deux systèmes complémentaires.

\subsubsection{Système en boucle fermée.}

Il s'agit ici de la technique utilisée dans les casques anti-bruit actifs [5]. Le signal de référence est directement fourni par le microphone de contrôle et le système fonctionne en boucle fermée. Le contrôleur est une cellule bi-quadratique analogique, dont le rôle est double : assurer une atténuation dans les basses fréquences tout en évitant les instabilités qui apparaissent en hautes fréquences. Par cette technique on a pu augmenter le coefficient d'absorption surtout dans les très basses fréquences [200, $800 \mathrm{~Hz}]$.

3.3.2 Système avec détection amont.

Le signal de référence est cette fois fourni par un microphone placé juste en amont de l'échantillon. Une évaluation de la fonction de transfert optimale du contrôleur nous a permis de constater qu'elle pouvait être réalisée sur une large gamme de fréquences par une cellule bi-quadratique. Ainsi le coefficient d'absorption a pu être ramené à 1 sur la gamme $500-1400 \mathrm{~Hz}$ (figure 7).

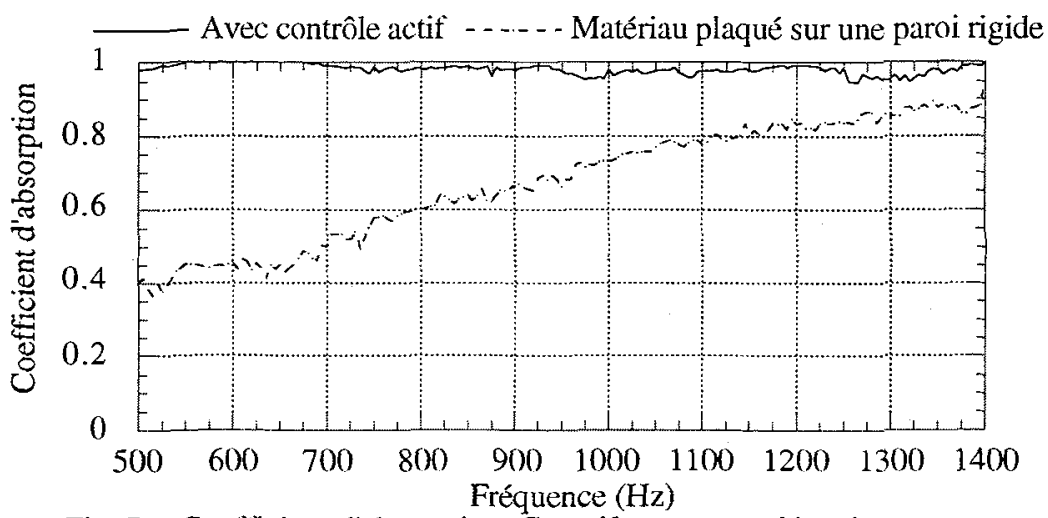

Fig. 7. - Coefficient d'absorption. Contrôle avec une détection amont

\section{CONCLUSIONS ET PERSPECTIVES}

Nous avons présenté ici une méthode alliant absorptions passive et active qui nous a permis de réaliser des parois très absorbantes, pour peu que les conditions suivantes soient respectées:

- l'épaisseur du matériau poreux utilisé doit être correctement choisie

- le système de contrôle actif réalise une minimisation de pression sur la face arrière du matériau.

Lorsqu'un tel absorbant est mis en place sur la paroi latérale d'un conduit, on a pu mettre en évidence par des mesures intensimétriques, une réduction de l'énergie acoustique au passage. Les essais en incidence oblique ou même aléatoire seront poursuivis de façon à constituer de véritables damiers d'efficacités spécifiques, réalisées par des systèmes de contrôle multivoies.

\section{REFERENCES}

[1] Delany M. E., Bazley E. N., "Acoustical Properties of fibrous materials", Appli. Acoust 3, 1970, p. $105-116$

[2] Allard J. F., "Propagation of sound in porous media : modelling sound absorbing materials", Elsevier Applied Science, 1993.

[3] Chung J. Y., Blaser D. A., "Transfer function method of measuring in-duct acoustic properties", J. Acoust. Soc. Am., 68, n³, 1980, p. 907-922.

[4] Thenail D., Galland M. A., "Development of an anechoidal boundary", Proc. Idee-Force EUR'ACOUSTICS, W-4, Ecole Centrale de Lyon, 1992.

[5] Carme C. "Absorption acoustique active dans les cavités auditives", Acustica, 66, n 5, p. 233-246, 1988. 